\title{
Heterologous expression of pathogen-specific genes ligA and ligB in the saprophyte Leptospira biflexa confers enhanced adhesion to cultured cells and fibronectin
}

Cláudio Pereira Figueira', Julio Croda ${ }^{1,6}$, Henry A Choy ${ }^{2,3}$, David A Haake ${ }^{2,3}$, Mitermayer G Reis ${ }^{1}$, Albert I Ko ${ }^{1,4}$ and Mathieu Picardeau ${ }^{*}$

\begin{abstract}
Background: In comparison to other bacterial pathogens, our knowledge of the molecular basis of the pathogenesis of leptospirosis is extremely limited. An improved understanding of leptospiral pathogenetic mechanisms requires reliable tools for functional genetic analysis. Leptospiral immunoglobulin-like (Lig) proteins are surface proteins found in pathogenic Leptospira, but not in saprophytes. Here, we describe a system for heterologous expression of the Leptospira interrogans genes ligA and ligB in the saprophyte Leptospira biflexa serovar Patoc.

Results: The genes encoding LigA and LigB under the control of a constitutive spirochaetal promoter were inserted into the L. biflexa replicative plasmid. We were able to demonstrate expression and surface localization of LigA and LigB in L. biflexa. We found that the expression of the lig genes significantly enhanced the ability of transformed L. biflexa to adhere in vitro to extracellular matrix components and cultured cells, suggesting the involvement of Lig proteins in cell adhesion.

Conclusions: This work reports a complete description of the system we have developed for heterologous expression of pathogen-specific proteins in the saprophytic L. biflexa. We show that expression of LigA and LigB proteins from the pathogen confers a virulence-associated phenotype on L. biflexa, namely adhesion to eukaryotic cells and fibronectin in vitro. This study indicates that L. biflexa can serve as a surrogate host to characterize the role of key virulence factors of the causative agent of leptospirosis.
\end{abstract}

\section{Background}

The genus Leptospira belongs to the order Spirochaetales and includes both saprophytic and pathogenic members, such as Leptospira biflexa and L. interrogans, respectively. Leptospirosis is the most widespread zoonosis worldwide, with more than one million cases annually $[1,2]$. Rodents are the principle reservoir of infections occurring in humans, resulting from renal tubular colonization and urinary excretion of the bacterium [3]. Humans are usually infected through water that is contaminated with the urine of animal reservoirs.

\footnotetext{
* Correspondence: mpicard@pasteur.fr

${ }^{5}$ Institut Pasteur, Unité de Biologie des Spirochètes, 28 rue du docteur Roux, 75724 Paris Cedex 15, France

Full list of author information is available at the end of the article
}

This increasingly common disease primarily occurs in rural environments and poor urban centres subject to frequent flooding. A major barrier to developing effective control of the disease has been our limited understanding of the biology of the bacterium. One of the reasons for this is the slow growth of pathogenic leptospires with a generation time of approximately 20 hours; colonies can take up to 4 weeks to appear on solid medium [4]. Furthermore, there are fewer tools for genetic studies of pathogenic leptospires than are available for many other bacterial pathogens. Tools for genetic manipulation of the saprophyte L. biflexa have been developed in recent years [4]. This work has significantly improved the feasibility of manipulating genes in pathogenic strains. For instance, we first developed systems

C Biomed Central

(c) 2011 Figueira et al; licensee BioMed Central Ltd. This is an Open Access article distributed under the terms of the Creative Commons Attribution License (http://creativecommons.org/licenses/by/2.0), which permits unrestricted use, distribution, and reproduction in any medium, provided the original work is properly cited. 
for targeted mutagenesis and random transposon mutagenesis in the saprophyte L. biflexa and then applied these approaches in the pathogen L. interrogans [5-7]. However, the introduction of exogenous genetic information into pathogenic strains by electroporation [8] or conjugation [9] is still hindered by poor transformation efficiencies. In addition, there is no replicative plasmid vector available for pathogenic Leptospira strains. Further development and improvement of genetic tools is therefore necessary for functional analysis of leptospiral virulence factors.

High-molecular-weight leptospiral immunoglobulinlike repeat (Lig) proteins were previously identified as putative virulence factors in pathogenic Leptospira spp. [10-12]. This family of three proteins - LigA, LigB and LigC - belongs to the superfamily of bacterial immunoglobulin (Ig)-like (Big) repeat domain proteins which includes virulence determinants such as intimin from enteropathogenic Escherichia coli and invasin from Yersinia pseudotuberculosis [10]. This superfamily appears to mediate pathogen-host cell interactions, such as invasion and host cell attachment, during infection. Several studies recently showed that recombinant Lig proteins can mediate in vitro interaction with fibronectin, fibrinogen, collagen, laminin, tropoelastin, and elastin [13-15]. Fibronectin-binding sites have also been identified in LigB $[14,16,17]$ and fibronectin-binding activity was shown to be modulated by calcium [18]. In addition, lig genes are up-regulated at physiological osmolarity [52] and encode surface-exposed proteins that are strongly recognized by sera from human leptospirosis patients $[11,19,20]$. Lig proteins are also protective antigens in animal models of leptospirosis [10,21-25]. Taken together, these data suggest that Lig proteins are major virulence factors and may contribute to the pathogen's ability to attach to host tissues during infection. However, additional research is essential to understanding how lig gene expression modifies this phenotype. We recently showed that the absence of LigB does not lead to a loss of virulence and colonization in the acutely- and chronically- infected animal models [6]. This may be due to functional redundancy of other surface-exposed proteins, including LigA, in the bacterium.

Despite the large evolutionary distance between the pathogenic and non-pathogenic species, we have shown that the Leptospira genus shares a core of approximately 2000 genes, including those encoding the relevant export pathways [26]. The saprophyte L. biflexa could therefore represent a good cloning host for the functional analysis of genes from poorly transformable pathogenic Leptospira.

In this study, we used the non pathogen $L$. biflexa serovar Patoc as a surrogate host to characterize the role of LigA and LigB in leptospiral interactions with eukaryotic cells and key host extracellular matrix proteins.

\section{Results}

\section{Expression of LigA and LigB in L. biflexa}

The saprophyte $L$. biflexa can be transformed at high rates with plasmids based on the LE1 replication origin, using kanamycin, spectinomycin, or gentamicin resistance as the selectable marker $[8,27,28]$. We chose the spectinomycin-resistant plasmid, pSLe94, as the backbone for our system: this shuttle plasmid containing the LE1 partition genes is stably maintained in L. biflexa in the absence of antibiotic selection [27]. Flagellin-encoding genes are usually both constitutively and strongly expressed. In addition, it has been reported that a kanamycin resistant cassette driven by the Borrelia burgdorferi flgB promoter is strongly expressed in B. burgdorferi [29] and in L. biflexa [4]. We therefore used the $f \lg B$ promoter from $B$. burgdorferi to allow strong and stable expression of LigA and LigB proteins in L. biflexa serovar Patoc (further indicated as Patoc). The genes encoding LigA and LigB under the control of the $f \lg B$ promoter were inserted into the $L$. biflexa replicative plasmid (Figure 1A). The Patoc wild-type (wt) strain was then electrotransformed by pSLePFligA and pSLePFligB, and the spectinomycin-resistant transformants were further analyzed. Lig expression by the lig-transformed Patoc strains was verified by Western blot analysis, which showed levels of protein comparable to the production by a low in vitro-passaged L. interrogans virulent strain (i.e. less than 10 in vitro passages). However, blots of the $\operatorname{lig} B$ transformant showed partial degradation of LigB (Figure 1B). The Patoc wt, ligA, and ligB strains had similar cell growth kinetics in EMJH liquid medium, indicating that the expression of the heterologous proteins did not affect cell growth (data not shown).

\section{Surface localization of LigA and LigB in L. biflexa}

LigA and LigB proteins have been shown to be surfaceexposed proteins in pathogenic Leptospira strains [11]. This was confirmed in this study with antibodies against LigA and LigB (see additional file 1: surface immunofluorescence assays in L. interrogans). Immunofluorescence studies found that antisera to LigA and LigB did not label the surface of the Patoc wt strain but did label the surface of the ligA- and ligB-transformed Patoc (Figure 2). The surface immunofluorescence binding assay specifically detected surface-exposed components because antiserum to whole bacteria labelled intact Patoc wt, Patoc ligA, and Patoc ligB whereas antisera to cytoplasmic heat-shock protein GroEL did not label live leptospires but was able to bind to permeabilized leptospires. LigA and LigB therefore appear to be surface- 


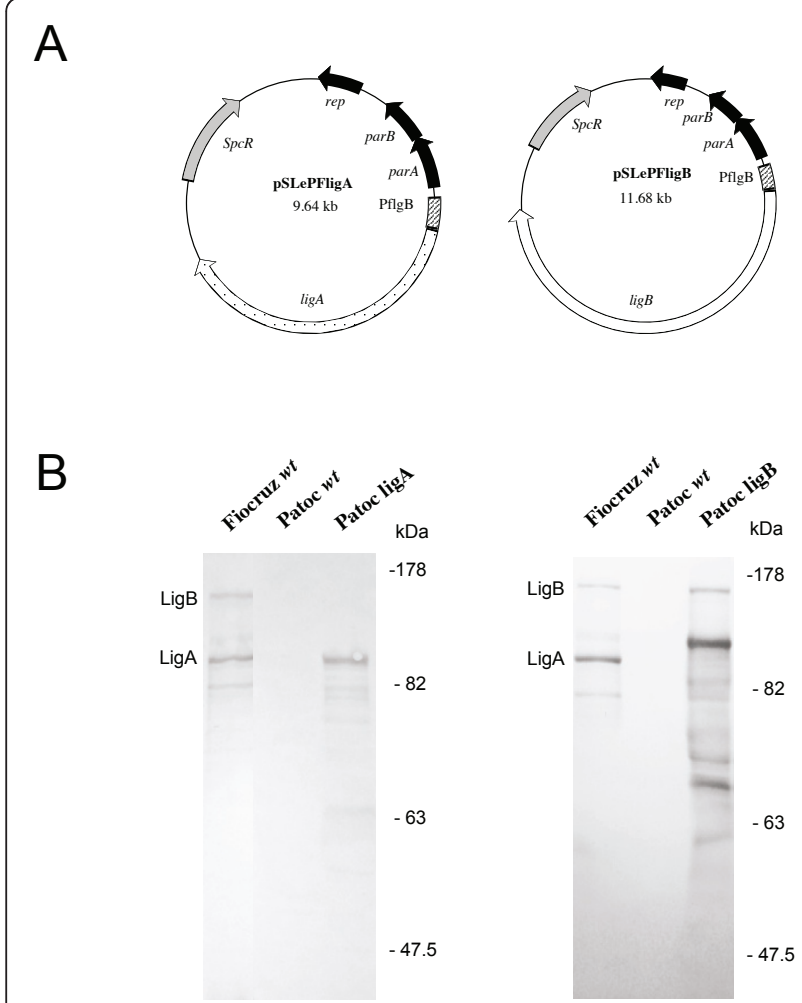

Figure 1 LigA and LigB expression in L. biflexa. A. Schematic diagram of plasmid constructs used to express constitutively LigA and LigB. The determinants for replication in L. biflexa (parAB and rep), as well as a spectinomycin (SpcR)- resistance cassette is indicated. B. Western blot of whole-cell lysates of $L$. interrogans serovar Copenhageni strain Fiocruz L1-130 (Fiocruz wt), L. biflexa serovar Patoc strain Patoc 1 (Patoc wt), and L. biflexa serovar Patoc strain Patoc 1 electrotransformed with pSLEPFligA (Patoc ligA) and pSLEPFligB (Patoc ligB) obtained by using LigA/B antiserum. The positions of standard molecular mass markers (in kilodaltons) are indicated on the left.

exposed when expressed in Patoc transformants carrying plasmid constructs pSLePFligA and pSLePFligB, respectively (Figure 2).

Host cell adhesion and translocation of lig-transformed $L$. biflexa

Interactions of Patoc wt, Patoc ligA, and Patoc ligB strains with mammalian host cells were assayed by examining adherence of leptospires to MDCK cells and translocation of leptospires across polarized MDCK cell monolayers. Adherence of L. interrogans strain Fiocruz L1-130 and Patoc ligA, but not Patoc wt and Patoc ligB, to MDCK cells was found to significantly increase in a time-dependent manner in two experiments (Figure 3). After a $240 \mathrm{~min}$ incubation period, approximately four times more Patoc ligA adhered to MDCK cells than Patoc wt and Patoc ligB. The number of adherent Patoc ligA leptospires per cell at $240 \mathrm{~min}$ incubation point was comparable ( 0.23 and 1.02 in experiments 1 and 2 , respectively) to that observed for the pathogenic $L$. interrogans strain Fiocruz L1-130 (0.16 and 0.73 in experiments 1 and 2, respectively).

Patoc ligA and ligB strains did not demonstrate enhanced ability to translocate across MDCK monolayers in comparison with Patoc wt in three experiments (representative experiment in Figure 4). As reported previously [30], we found that a small proportion $(<1 \%)$ of Patoc wt was able to translocate across MDCK monolayers after a $240 \mathrm{~min}$ incubation period. Proportions of translocating leptospires recovered from the lower transwell chamber were not significantly different between Patoc wt and Patoc ligA and ligB during the assay's time course (Figure 4). In contrast, > $6 \%$ of the inoculum of pathogenic L. interrogans strain Fiocruz L1-130 was recovered in the lower chamber after $240 \mathrm{~min}$ of incubation (Figure 4). As previously reported [30], recovery of L. interrogans strain Fiocruz L1-130 was not associated with significant alterations in the TER (Figure 4), indicating that disruption of tight junctions of the monolayers did not occur during the translocation process. Together these findings indicate that whereas expression of LigA in the saprophyte Patoc was associated with an enhanced host cell adherence phenotype similar to that observed with pathogenic leptospires, it did not impart the ability to efficiently invade and translocate across polarized host cell monolayers.

\section{Enhanced adhesion to fibronectin and laminin by lig- transformed $L$. biflexa}

Lig recombinant proteins have been shown to recognize in vitro host extracellular matrix proteins $[13,14]$. The introduction of the ligA or ligB gene from pathogenic $L$. interrogans into the nonpathogenic saprophyte $L$. biflexa enhanced the adhesion of the latter to the mammalian host protein fibronectin (Figure 5A). The lig transformants bound to both plasma and cellular fibronectin approximately two-fold better than the Patoc wild-type strain (2.0-fold average for 1.7- to 2.3-fold range in four independent determinations for the $\operatorname{lig} A$ cells; 2.2 -fold average from 1.5- to 3.1-fold in five measurements with $\operatorname{lig} B$ ). The wild-type cells showed non-Lig-mediated adherence to fibronectin, which may reflect the ability of the saprophyte to interact with related proteins in decaying material that it encounters in the environment. Transformation with the lig genes also increased laminin binding 1.2-fold in comparison to the Patoc wild-type strain (Figure $5 \mathrm{~B}$ ). However, the $\operatorname{lig} A$ or $\operatorname{lig} B$ cells did not appear to bind elastin better than wild-type cells, and all three strains interacted weakly with type I and type IV collagen (Figure 5B). 


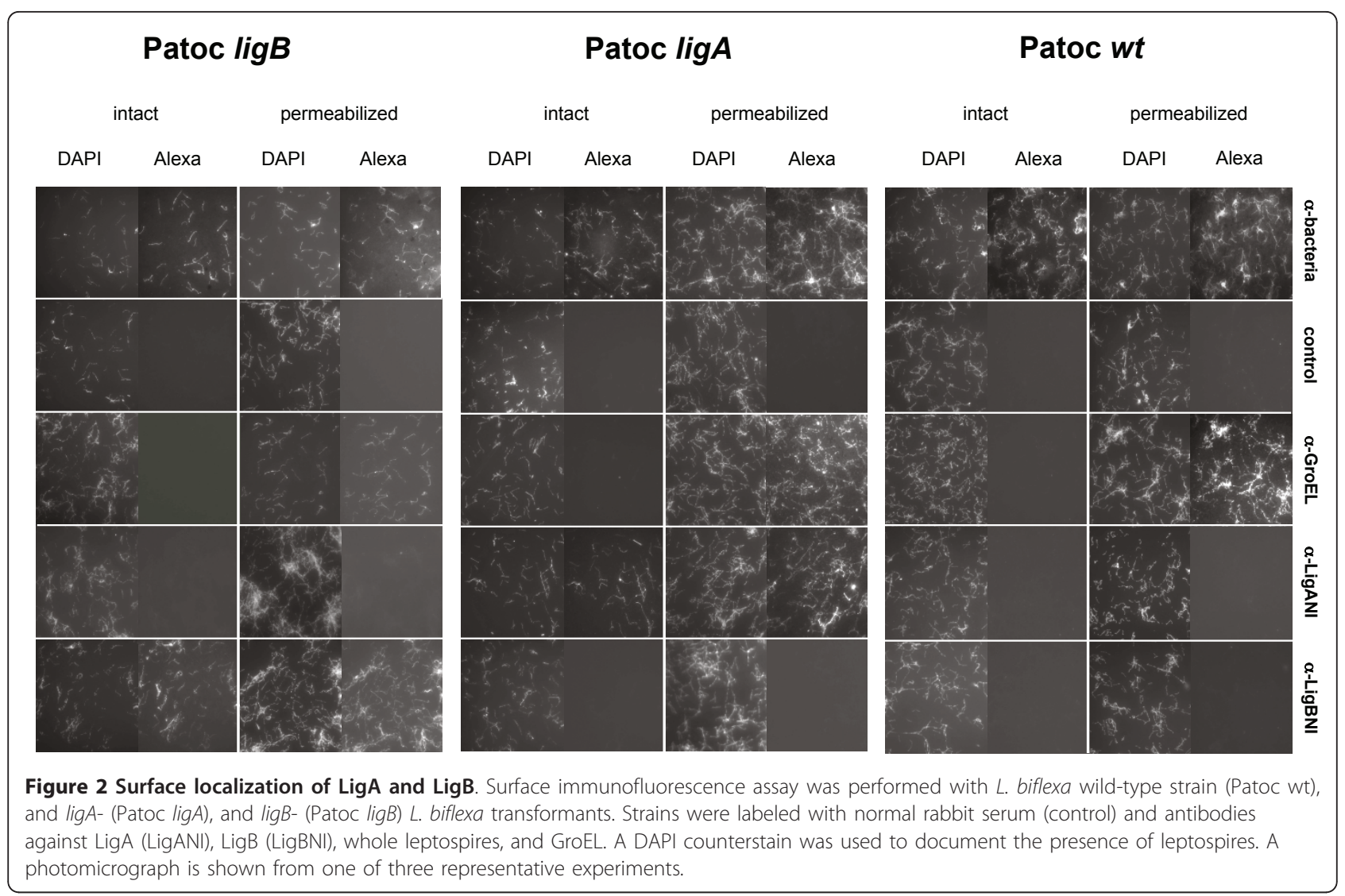

\section{Discussion}

The lack of genetic tools has hampered molecular analyses of putative virulence factors in pathogenic Leptospira spp. In this work, we showed for the first time that pathogen-specific proteins can be expressed in a saprophytic Leptospira and that expression of such proteins
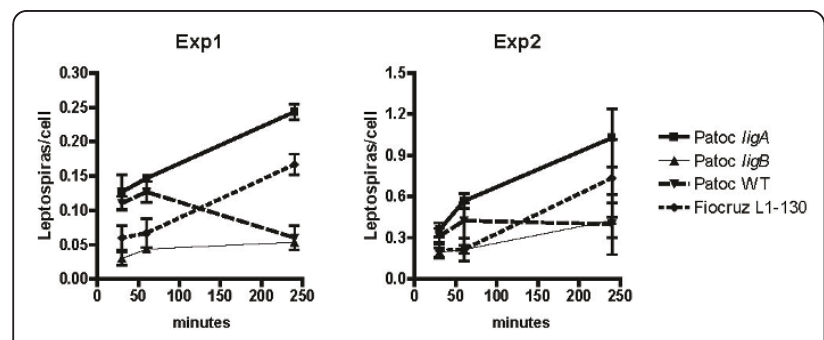

Figure 3 Association of L. biflexa transformants with MDCK monolayers. Adhesion of MDCK epithelial cells with $L$. interrogans (L1-130), L. biflexa wild-type strain (wt), and ligA- (ligA), and ligB(ligB) L. biflexa transformants. Results were determined after 30, 60, and 240 minutes exposure, followed by extensive washing of nonadherent bacteria. The bars show the mean number of bacteria associated per host cell \pm standard deviation carried out in 10 random fields in two independent experiments. The numbers of adherent leptospires/cell between the L. biflexa wild-type strain and the ligA- and ligB- L. biflexa transformants were statiscally different at 240 minutes $(P<0.05)$. are accompanied by an in vitro virulence associated phenotype. The approach used in this study demonstrates that the fast-growing non pathogenic species $L$. biflexa serves a model for examining pathogenetic mechanisms of $L$. interrogans. In contrast to L. biflexa, data obtained when $E$. coli was used as a surrogate host revealed that

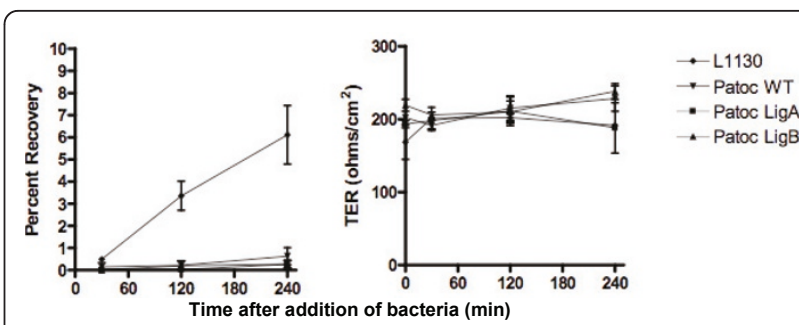

Figure 4 Translocation assays. Percent recovery of leptospires (left) and TER (right) after inoculation of polarized MDCK cell monolayers with L. interrogans Fiocruz L1-130 (L1-130), L. biflexa wild-type strain (Patoc wt), and ligA-(Patoc ligA), and ligB-(Patoc ligB) L. biflexa transformants. Bacteria were inoculated in the upper chamber of MDCK cell monolayer transwell chambers. Translocating bacteria was quantified by counting bacteria in the lower chamber. Assays were performed at 30, 120, and 240 minutes (min) after addition of bacteria. The assays were performed in triplicate, and results are expressed as mean $\pm S D$. The findings of a representative experiment, among three which were performed, are shown. 


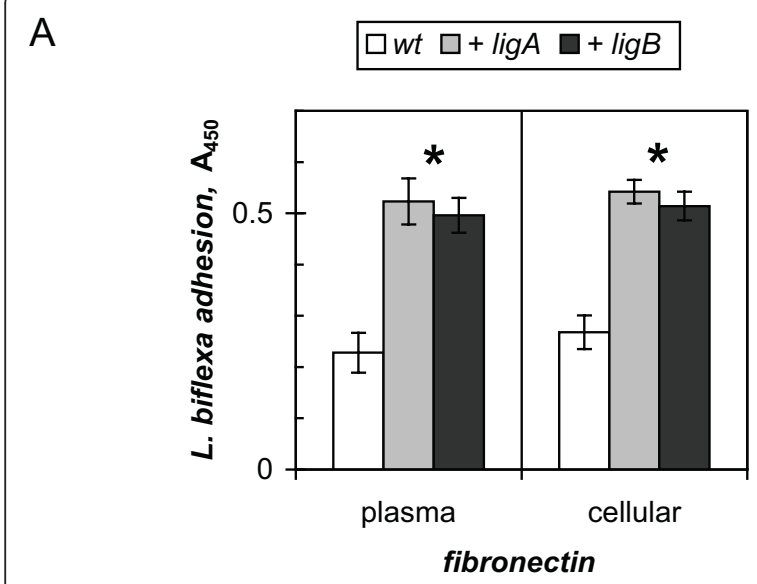

B

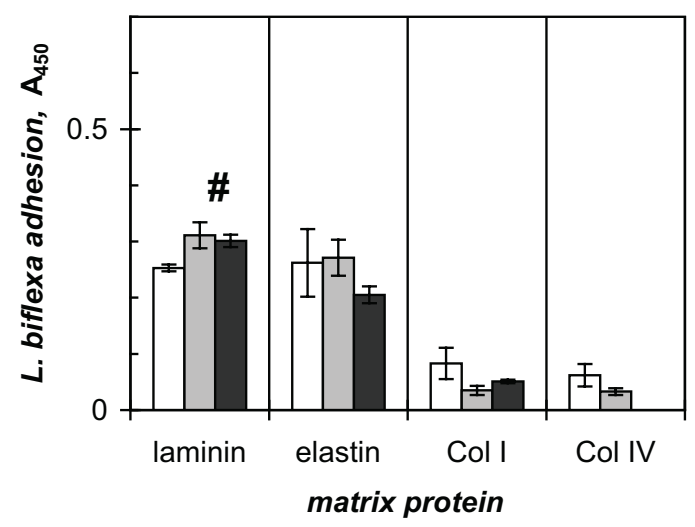

Figure 5 Binding of L. biflexa transformants to extracellular matrix components. A. Fibronectin binding assay was performed with L. biflexa wild-type strain $(w t)$, and ligA- $(+\operatorname{lig} A)$, and ligB- $(+\operatorname{lig} B)$ transformed $L$. biflexa. The means and standard deviations of triplicates from a representative of more than three independent experiments are shown, with statistical significance at $P<0.01\left(^{*}\right)$. B. Laminin, elastin, and collagen type I ( $\mathrm{Co} / \mathrm{I})$ and type IV ( $\mathrm{Co} / \mathrm{M}$ ) binding was measured as in $\mathrm{A}$. with $\mathrm{P}<0.05$ (\#).

most of the spirochetal promoters functioned poorly in this genetic background. Even when leptospiral proteins are expressed in E. coli, many are found to be insoluble. An additional consideration is that a number of leptospiral proteins undergo post-translational modifications that may not occur in Gram negative bacteria [31].

In this study, the L. interrogans LigA and LigB lipoproteins were expressed and exposed on the surface of L. biflexa cells. However, the ligB-transformed L. biflexa produced almost no full length LigB protein. This suggests that $L$. biflexa is an appropriate surrogate host for expression of at least some $L$. interrogans outer membrane proteins [26]. These experimental results confirm genome sequence analyses indicating that most of the known protein export and processing systems of
L. interrogans and L. biflexa are highly conserved [26]. Surface localization of Ligs in the model bacterium $L$. biflexa presents a unique opportunity to study the translocation of lipoproteins through leptospiral membranes. Further study could, for instance, include the analysis of the leptospiral lipobox which is distinct from the motifs of $E$. coli and other gram-negative bacteria. For example, the leptospiral surface lipoprotein, LipL41 was not efficiently expressed in $E$. coli until its lipobox was altered to mimic that of murein lipoprotein [32]. Analysis of leptospiral lipobox sequences indicates that most leptospiral lipoproteins would be anticipated to not be processed correctly in E. coli [33].

Bacterial adhesion is a crucial step in the infectious process.

Among members of the superfamily of bacterial immunoglobulin (Ig)-like (Big) proteins, previous studies have demonstrated that in comparison to the wild type strain, an intimin-deficient enteropathogenic E. coli strain is defective in adherence to cultured cells and in intestinal colonization [34]. In Y. enterocolitica, an invasin mutant was impaired in its ability to translocate the intestinal epithelium [35]. By contrast, we found that a L. interrogans $\operatorname{lig} B^{-}$mutant retained its virulence and ability to adhere to MDCK cells [6]. This may be due to functional redundancy of other Lig proteins such as LigA. To determine the function of lig genes in pathogens, it may therefore be necessary to knock-out multiple genes, which would not be feasible in pathogenic Leptospira strains.

This study is a complete description of our approach for heterologous expression of pathogen-specific proteins in the saprophyte, L. biflexa serovar Patoc, resulting in the acquisition of virulence-associated phenotype. We demonstrate that Patoc ligA is able to adhere to epithelial cells in a time-dependent fashion, comparable to the pathogen L. interrogans. In addition, levels of binding of Patoc ligA and Patoc ligB to fibronectin and laminin were significantly higher in comparison to Patoc wt. However, lig transformants did not appear to bind collagens (type I and IV) or elastin better than wild-type cells. Analysis of Patoc ligA and Patoc ligB suggests that the Lig proteins are not sufficient for the efficient translocation of the bacteria across the cell monolayers, a characteristic that distinguishes leptospiral pathogens from saprophytes [30]. This result suggests that invasion is a more complex process than adherence and may require additional properties unique to leptospiral pathogens. In other words, invasion of cellular monolayers may require a stepwise adherence process involving interactions with a series of host ligands. Recently, we described enhanced fibrinogen binding of L. biflexa expressing LigA and LigB using the same plasmid constructs described here as part of a general examination 
of Lig-fibrinogen interactions [36], validating the relevance of our heterologous expression system.

Studies involving recombinant proteins, including LigA and LigB, have revealed a number of proteins that bind to extracellular matrix proteins [37-43]. Whether the functions of these putative adhesins are overlapping or synergistic in the interactions of leptospiral cells with eukaryotic cells or monolayers is unknown. LigA and LigB proteins contain related yet distinct Big domains that may share redundant function [13-15]. For example Choy et al demonstrated that portions of both LigA and LigB proteins bind fibronectin in vitro [13]. Thus the function of LigB can be substituted to varying extents by other lipoproteins, including LigA, which may play a role in hostcell interactions. The use of L. biflexa as a surrogate host enables functional studies of virulence factors in isolation without interference from activities of competing or redundant outer membrane proteins. Further studies expressing distinct regions of LigA and LigB in L. biflexa are required to understand the precise role of each domain in the binding of components of the extracellular matrix.

L. interrogans is an invasive pathogen that can adhere and translocate through host cells $[30,44]$. In contrast to the increased adherence of the ligA-transformed $L$. biflexa strain to MDCK renal cells, the ligB transformants did not exhibit enhanced attachment to the eukaryotic cells following four hours of incubation. This may be due to the partial degradation of LigB observed in these transformants by Western blots (Figure 1B). In contrast, we found that both ligA- and ligB-transformed $L$. biflexa bound fibronectin in significantly greater numbers than wild-type L. biflexa in a solid-phase assay format (Figure 5A). The large remaining LigB fragment appears slightly larger than intact LigA, suggesting that the degraded LigB may comprise the immunoglobulinlike repeats containing the fibronectin-binding domain [13]. These findings suggest that lig-mediated host cell adhesion may involve receptors in addition to fibronectin. The expression and localization of fibronectin and its binding integrins as well as other components of extracellular matrix in MDCK cells are dependent on culture conditions $[45,46]$. It is possible that the large proteolytic fragment of LigB remaining with the $\operatorname{lig} B$ transformants retains the fibronectin-binding region but has lost sequences mediating the interaction of LigB with a different and distinct renal cell receptor. Further studies with lig transformants could include analyzing lig-mediated host cell adhesion by using additional cell lines representing different species and cell types.

\section{Conclusion}

In conclusion, by using L. biflexa as a surrogate host, we have shown that Lig proteins are factors involved in the attachment to fibronectin, fibrinogen, and laminin and to host cells and can act as microbial surface components recognizing host extracellular matrix proteins. Although important advances in the genetic system of the pathogen $L$. interrogans have been made in the last years [5,7], this bacterium remains poorly transformable and few mutants have been fully characterized [3]. We believe that $L$. biflexa can serve as a model bacterium for investigating the function of additional leptospiral pathogenesis mechanisms. Genetic studies in L. biflexa should provide information about the roles of key components in the pathogenesis of leptospirosis.

\section{Methods}

\section{Bacterial strains and culture conditions}

Leptospires were cultivated in liquid EllinghausenMcCullough-Johnson-Harris (EMJH) medium $[47,48]$ or on $1 \%$ agar plates at $30^{\circ} \mathrm{C}$ and counted in a PetroffHausser counting chamber (Fisher Scientific). The saprophyte Leptospira biflexa serovar Patoc strain Patoc I and the pathogen L. interrogans serovar Copenhageni strain Fiocruz L1-130 were used in this study. E. coli was grown in Luria-Bertani (LB) medium. When appropriate, spectinomycin or kanamycin was added to culture medium at the final concentration of $40 \mu \mathrm{g} / \mathrm{ml}$.

\section{Plasmid constructions}

The Borrelia burgdorferi flgB promoter was amplified with PflgA (5'-TAATACCCGAGCTTCAAGGAAG-3') and PflgB (5'-AACATATGGAAACCTCCCTC-3') and cloned into pCR2.1 (Invitrogen) to generate plasmid pCRPromFlgB. The $\operatorname{lig} A$ and $\operatorname{lig} B$ genes were amplified with flanking $N d e I$ and $X h o I$ sites, using primer pairs LANF (5'-GGGAATTCCATATGAAGAAAATATTTT GTATTTCG-3') - LAXR (5' CGGCTCGAGTTATTAT GGCTCCGTTTTAATAGAGG-5') and LBNF (5'-GGG AATTCCATATGAAGAAAATATTTTGTATTTCG-5') - LBXR (5'-CGGCTCGAGTTATTATTGATTCTGTT GTCTGT-3'), respectively, from genomic DNA of L. interrogans serovar Copenhageni strain Fiocruz L1-130. Amplified lig genes were then digested with NdeI and $X h o I$ restriction enzymes, purified, and inserted between the corresponding restriction sites of pCRPromFlgB to generate pCRPflgBLigA and pCRPflgBLigB, respectively. The DNA fragment containing $\operatorname{Prom}_{f l g B} \operatorname{ligA}(4183 \mathrm{bp})$ and $\operatorname{Prom}_{f l g B}$ ligB (6188 bp) were released from plasmids pCRPflgBLigA and pCRPflgBLigB by SpeI and XbaI digestion, then blunt-ended, and cloned into the PvuII restriction site of the E. coli-L. biflexa shuttle vector pSLe94 [49] to generate pSLePFligA and pSLePFligB (Figure 1). Plasmid constructs were verified by nucleotide sequencing.

lig-transformed L. biflexa

L. biflexa was prepared for transformation as previously described [4]. In brief, L. biflexa was grown at $30^{\circ} \mathrm{C}$ 
until the optical density reached 0.4 at $420 \mathrm{~nm}$. Bacteria were collected by centrifugation at room temperature and washed by resuspension in deionized water followed by centrifugation. After removing the supernatant fluid, the bacteria were resuspended with deionized water to a final concentration of around $5 \times 10^{10}$ cells $/ \mathrm{ml}(100 \times$ concentration). $100 \mu \mathrm{l}$ of the suspended bacteria were added to the plasmid DNA, and the DNA-bacteria mixture was added to chilled electroporation cuvettes with a $0.2 \mathrm{~cm}$ gap. The cuvette was placed in the electroporation unit (Bio-Rad Gene Pulser II) and subjected to electroporation at a setting of $1.8 \mathrm{kV}, 25 \mu \mathrm{F}$, and $200 \Omega$. After adding $1 \mathrm{ml}$ of $\mathrm{EMJH}$, the bacteria were transferred to a $15 \mathrm{ml}$ Falcon tube and incubated for 24 hours at $30^{\circ} \mathrm{C}$ with shaking. The culture $(0.2 \mathrm{ml})$ was plated onto EMJH plates containing $40 \mu \mathrm{g} / \mathrm{ml}$ of spectinomycin and incubated at $30^{\circ}$ for 10 days. Colonies were inoculated into liquid EMJH containing $40 \mu \mathrm{g} / \mathrm{ml}$ spectinomycin. L. biflexa transformants were maintained by serial passage in the liquid medium.

\section{Western Blot}

Exponential phase cultures of $L$. biflexa Patoc wild-type, Patoc ligA, Patoc ligB, and L. interrogans Fiocruz strains were washed, resuspended in PBS and solubilized in $62.5 \mathrm{mM}$ Tris hydrochloride (pH 6.8)-10\% glycerol-5\% 2 -mercaptoethanol-2\% sodium dodecyl sulfate. A $20 \mu \mathrm{l}$ volume of crude extracts containing $2 \times 10^{8}$ bacteria/ per well was resolved by $8 \%$ sodium dodecyl sulfate (SDS)-polyacrylamide gel electrophoresis using a discontinuous buffer system. After transfer to nitrocellulose membranes, immunoblots were blocked in $0.05 \mathrm{M}$ Trisbuffered saline (pH 7.4)-0.05\% ( $\mathrm{vol} / \mathrm{vol}$ ) Tween 20 with $5 \%$ (wt/vol) nonfat dry milk. The blots were washed, incubated for $1 \mathrm{~h}$ at room temperature with a 1,000-fold dilution of mouse ascites containing MAb to the $\operatorname{LigB}$ identical repeat region $(\operatorname{Lig} A / B)[6]$ and probed with goat anti-mouse conjugated to alkaline phosphatase (Sigma). Immunoblots were developed in a nitroblue tetrazolium-5-bromo-4-chloro-3-indolylphosphate (BCIP) solution (Bio-Rad).

\section{Localization of LigA/LigB by immunofluorescence}

We evaluated the localization of LigA and LigB by performing immunofluorescence labeling according to a modified protocol of Cullen et al. [50]. Suspensions of $10^{7}$ live leptospires in $10 \mu \mathrm{l}$ of PBS were placed onto poly-L-lysine-coated slides (Sigma-Aldrich) for $1 \mathrm{~h}$ in a humidified chamber for adherence of the leptospires. In experiments in which the bacteria were permeabilized prior to incubation with antibody, slides were incubated with cold methanol for $10 \mathrm{~min}$ at $-20^{\circ} \mathrm{C}$, followed by two washes with PBS. Blocking with $1 \%$ bovine serum albumin (Sigma-Aldrich) (PBS-BSA) for 20 min was performed before incubation for $1 \mathrm{~h}$ at $37^{\circ} \mathrm{C}$ with normal rabbit serum, rabbit hyperimmune antisera to whole extracts of $L$. interrogans serovar Icterohaemorrhagiae strain RGA, LigB non-identical region (LigBNI), and LigA non-identical region (LigANI) [6], and rat antiserum to GroEL, which were diluted 1:100 in PBS-BSA. The slides were washed gently with PBS-BSA and incubated with goat anti-rabbit IgG antibodies conjugated to Alexa dye (Molecular Probes) or goat anti-rat IgG antibodies conjugated to fluorescein isothiocyanate (Jackson ImmunoResearch Laboratories) for $1 \mathrm{~h}$ at $37^{\circ} \mathrm{C}$. The slides were washed twice with PBS-BSA and incubated with $1 \mu \mathrm{g} / \mathrm{ml}$ DAPI (Molecular Probes) for $1 \mathrm{~h}$ at room temperature. Slides were then washed, then mounted in anti-fading solution (Prolong-Molecular Probes) and visualized by fluorescence microscopy (Olympus BX51).

\section{Adhesion and translocation assays with MDCK cells}

Madin Darby canine kidney (MDCK) cells were grown in Dulbecco's Modified Eagle's Medium (DMEM) supplemented with $10 \%$ fetal bovine serum (Cultilab), $2 \%$ sodium bicarbonate, $25 \mathrm{mM}$ HEPES, and $5 \mathrm{mM}$ L-glutamine (Sigma) at $37^{\circ} \mathrm{C}$ in an atmosphere of $5 \% \mathrm{CO}_{2}$. MDCK cells were harvested by treating cell cultures with $0.05 \%$ trypsin and $0.02 \%$ EDTA in PBS. For adhesion assays, MDCK cells were plated onto 24-well plates in DMEM, containing 13-mm-diameter glass coverslips at $37^{\circ} \mathrm{C}$ in an atmosphere of $5 \% \mathrm{CO}_{2}$ until they were confluent. The number of MDCK cells in wells was determined by lysing cells with $0.1 \mathrm{M}$ citric acid containing $0.05 \%$ crystal violet (Sigma-Aldrich) and $1 \%$ Cetrimide (Sigma) [51], then the nuclei were counted in a hemacytometer. The cells were incubated with a suspension of Patoc wild-type, Patoc ligA, Patoc ligB and Fiocruz L1-130 strains in cell culture medium at the final bacteria: cell ratio of 10:1. Incubations were performed for periods of 30 to $240 \mathrm{~min}$. Prior to staining, the cells were washed three times in PBS to remove nonadherent bacteria and then fixed with cold methanol for $10 \mathrm{~min}$. An immunofluorescence assay was performed to detect adherent leptospires for which rabbit polyclonal antisera against whole extracts of $L$. interrogans strain RGA and goat anti-rabbit antibodies conjugated with Alexa488 (Molecular Probes) were used as first and second antibodies, respectively. DAPI and Alcian Blue were used to stain the nucleus and cytoplasm, respectively. The number of leptospires and MDCK cells was determined by examining ten highmagnification $(1000 \times)$ fields during fluorescence microscopy. All incubation points were performed in triplicate. The ANOVA test was used to determine statistically significant $(\mathrm{p}<0.05)$ differences between numbers of adherent leptospires/cell. We performed a translocation assay according to a protocol modified 
from that described by Barocchi et al [30]. MDCK cells at a concentration of $2 \times 10^{5}$ cells in $500 \mu \mathrm{l}$ of DMEM were seeded onto 12 -mm-diameter Transwell filter units with $3-\mu \mathrm{m}$ pores. Monolayers were incubated at $37^{\circ} \mathrm{C}$ in $5 \% \mathrm{CO}_{2}$ for 3 to 4 days with daily changes in media until the transmonolayer electrical resistance (TER) reached a range of 200 and $300 \Omega / \mathrm{cm}^{2}$, as measured with an epithelial voltohmmeter (EVOM, World Precision Instruments, Sarasota, Fla.). The TER for polycarbonate filters without cells was approximately $100 \Omega$ / $\mathrm{cm}^{2}$. The upper chamber of the transwell apparatus was inoculated with leptospires at a multiplicity of infection (MOI) of 100 by adding $500 \mu \mathrm{L}$ of bacteria which were resuspended in 1:2 v/v ratio of DMEM and EMJH media. Duplicate transwell chamber assays were performed for each leptospiral strain which were tested. Aliquots were removed from lower chamber $(100 \mu \mathrm{l})$ at 30,120 and $240 \mathrm{~min}$ and the number of leptospires was counted in triplicate by using the Petroff-Hausser chamber. The ability of leptospires to translocate MDCK polarized monolayers was determined by calculating the proportion of leptospires in the lower chamber in comparison to the initial inoculum for duplicate assays at each time point. The ANOVA test was used to determine significant differences in the proportions of translocating leptospires and TER values obtained during incubations with different leptospiral strains.

\section{ELISA for binding to extracellular matrix components}

The adhesion of live L. biflexa strains to immobilized fibronectin was measured with an ELISA. Two to three $\times 10^{8}$ cells in serum-free EMJH or medium alone was incubated at $30^{\circ} \mathrm{C}$ for $1 \mathrm{~h}$ in a microtiter well pre-coated with $1 \mu \mathrm{g}$ of fibronectin (from human plasma or foreskin fibroblasts, Sigma-Aldrich), collagen type I (bovine skin, Sigma-Aldrich), collagen type IV (human placenta, Sigma-Aldrich), laminin (murine, Sigma-Aldrich), elastin (human skin, Elastin Products Company, Owensville, $\mathrm{MO}$ ), or left in PBS, pH7.2, overnight at $4^{\circ} \mathrm{C}$. Uncoated sites in the well were covered with Protein-Free Blocker (Thermo Scientific) before the addition of cells. Adherent cells were fixed with $4 \%$ formaldehyde (Thermo Scientific) at room temperature for $1 \mathrm{~h}$, tagged with a rabbit polyclonal antibody for intact L. biflexa (MyBioSource), and detected by spectrometry at $450 \mathrm{~nm}$ to measure the activity of horseradish peroxidase conjugated to a donkey antibody for rabbit IgG (GE Healthcare). Backgrounds from uncoated wells (PBS) and medium only were subtracted. Triplicate assays were done and statistically significant differences in adhesion were determined with one-way ANOVA compared to the wild-type cells.

\section{Additional material}

Additional file 1: surface immunofluorescence assays in $L$. interrogans. Immunofluorescence assays were performed with $L$ interrogans strain Fiocruz L1-130, which was labeled with normal rabbit serum (control) and antibodies against LigA (LigANI), LigB (LigBNI), GroEL, and LPS. Alexa- and fluorescein isothiocyanate-conjugated secondary antibodies were used to detect surface-bound antibodies. A DAPI counterstain was used to document the presence of leptospires. The photomicrograph show the results of one of three representative experiments.

\section{Acknowledgements}

This work was supported by the Institut Pasteur, Paris, France; the French Ministry of Research ANR-08-MIE-018, the Fiocruz-Pasteur Scientific Cooperation Agreement, Oswaldo Cruz Foundation (PDTIS RVR05), Brazilian National Research Council (INCTV), VA Medical Research Funds, and the National Institutes of Health (grants D43 TW00919, R01 Al34431 and U01 Al088752). This research was conducted by C.P. Figueira in partial fulfillment of the requirements for a Ph.D. from Goncalo Moniz Research Center, Oswaldo Cruz Foundation, Brazil.

\section{Author details}

${ }^{1}$ Oswaldo Cruz Foundation, Brazilian Ministry of Health, Gonçalo Moniz Research Center, Rua Waldemar Falcão, 121, 40295-001 Salvador, Bahia, Brazil. ${ }^{2}$ Veterans Affairs Greater Los Angeles Health Care System, Division of Infectious Diseases, 111F, 11301 Wilshire Blvd, Los Angeles, CA 90073, California, USA. ${ }^{3}$ Department of Medicine, University of California Los Angeles School of Medicine, Los Angeles, California, USA. ${ }^{4}$ Division of Epidemiology of Microbial Diseases, Department of Epidemiology and Public Health, Yale University School of Medicine, Division of Epidemiology of Microbial Disease, 319 LEPH - 60 College St., New Haven, CT 06510 New Haven, USA. ${ }^{5}$ Institut Pasteur, Unité de Biologie des Spirochètes, 28 rue du docteur Roux, 75724 Paris Cedex 15, France. ${ }^{6}$ Faculty of Health Sciences, Federal University of Grande Dourados, Brazil.

\section{Authors' contributions}

AIK, DAH, HAC, and MP conceived the study. JC generated the plasmid constructs. CPF performed immunofluorescence, adhesion, and translocation assays. HAC performed the fibronectin binding assays. CPF, AIK, DAH, HAC, $M G R$, and MP participated in data interpretation and manuscript preparation. All authors read and approved the manuscript.

Received: 21 March 2011 Accepted: 9 June 2011 Published: 9 June 2011

\section{References}

1. Bharti AR, Nally JE, Ricaldi JN, Matthias MA, Diaz MM, Lovett MA, Levett PN, Gilman RH, Willig MR, Gotuzzo E, VInetz JM: Leptospirosis: a zoonotic disease of global importance. Lancet Infect Dis 2003, 3:757-771.

2. Levett PN: Leptospirosis. Clin Microbiol Rev 2001, 14:296-326.

3. Ko Al, Goarant C, Picardeau M: Leptospira: the dawn of the molecular genetics era for an emerging zoonotic pathogen. Nat Rev Microbiol 2009, 7:736-747.

4. Louvel H, Picardeau M: Genetic Manipulation of Leptospira biflexa. Hoboken, N.J.: J. Wiley and Sons; 2007.

5. Bourhy P, Louvel H, Saint Girons I, Picardeau M: Random insertional mutagenesis of Leptospira interrogans, the agent of leptospirosis, using a mariner transposon. J Bacteriol 2005, 187:3255-3258.

6. Croda J, Figueira CP, Wunder EAJ, Santos CS, Reis MG, Ko Al, Picardeau M: Targeted mutagenesis in pathogenic Leptospira: Disruption of the ligB gene does not affect virulence in animal models of leptospirosis. Infect Immun 2008, 76:5826-5833.

7. Murray GL, Morel V, Cerqueira GM, et al: Genome-wide transposon mutagenesis in pathogenic Leptospira spp. Infect Immun 2009, 77:810-816.

8. Saint Girons I, Bourhy P, Ottone C, Picardeau M, Yelton D, Hendrix RW, Glaser $P$, Charon N: The LE1 bacteriophage replicates as a plasmid within Leptospira biflexa: construction of an L. biflexa-Escherichia coli shuttle vector. J Bacteriol 2000, 182:5700-5705. 
9. Picardeau M: Conjugative transfer between Escherichia coli and Leptospira spp. as a new genetic tool. Appl Environ Microbiol 2008, 74:319-322.

10. Koizumi $\mathrm{N}$, Watanabe $\mathrm{H}$ : Leptospiral immunoglobulin-like proteins elicit protective immunity. Vaccine 2004, 22:1545-1552.

11. Matsunaga J, Barocchi MA, Croda J, et al: Pathogenic Leptospira species express surface-exposed proteins belonging to the bacterial immunoglobulin superfamily. Mol Microbiol 2003, 49:929-945.

12. Palaniappan RU, Chang YF, Jusuf SS, et al: Cloning and molecular characterization of an immunogenic LigA protein of Leptospira interrogans. Infect Immun 2002, 70:5924-5930.

13. Choy HA, Kelley MM, Chen TL, Møller AK, Matsunaga J, Haake DA: Physiological osmotic induction of Leptospira interrogans adhesion: LigA and LigB bind extracellular matrix proteins and fibrinogen. Infect Immun 2007, 75:2441-2450.

14. Lin YP, Chang YF: A domain of the Leptospira LigB contributes to high affinity binding of fibronectin. Biochem Biophys Res Commun 2007, 362:443-448.

15. Lin YP, Lee DW, McDonough SP, Nicholson LK, Sharma Y, Chang YF: Repeated domains of leptospira immunoglobulin-like proteins interact with elastin and tropoelastin. J Biol Chem 2009, 284:19380-19391.

16. Lin YP, Chang YF: The C-terminal variable domain of LigB from Leptospira mediates binding to fibronectin. J Vet Sci 2008, 9:133-144

17. Lin YP, Greenwood A, Yan W, Nicholson LK, Sharma Y, McDonough SP, Chang YF: A novel fibronectin type III module binding motif identified on C-terminus of Leptospira immunoglobulin-like protein, LigB. Biochem Biophys Res Commun 2009, 389:57-62.

18. Lin YP, Raman R, Sharma Y, Chang YF: Calcium binds to leptospiral immunoglobulin-like protein, LigB, and modulates fibronectin binding. J Biol Chem 2008, 283:25140-24149.

19. Croda J, Ramos JG, Matsunaga J, Queiroz A, Homma A, Riley LW, Haake DA, Reis MG, Ko Al: Leptospira immunoglobulin-like proteins as a serodiagnostic marker for acute leptospirosis. J Clin Microbiol 2007, 45:1528-1534.

20. Srimanote $P$, Wongdeethai $N$, Jieanampunkul $P$, Samonkiert $S$, Leepiyasakulchai C, Kalambaheti T, Prachayasittikul V: Recombinant LigA for leptospirosis diagnosis and ligA among the Leptospira spp. clinical isolates. J Microbiol Methods 2008, 72:73-81.

21. Faisal SM, Yan W, Chen CS, Palaniappan RU, McDonough SP, Chang YF: Evaluation of protective immunity of Leptospira immunoglobulin like protein $A$ (LigA) DNA vaccine against challenge in hamsters. Vaccine 2008, 26:277-287.

22. Faisal SM, Yan W, McDonough SP, Chang YF: Leptospira immunoglobulinlike protein A variable region (LigAvar) incorporated in liposomes and PLGA microspheres produces a robust immune response correlating to protective immunity. Vaccine 2009, 27:378-387.

23. Palaniappan RU, McDonough SP, Divers TJ, Chen CS, Pan MJ, Matsumoto M, Chang YF: Immunoprotection of recombinant leptospiral immunoglobulin-like protein A against Leptospira interrogans serovar Pomona infection. Infect Immun 2006, 74:1745-1750.

24. Silva EF, Medeiros MA, McBride AJ, et al: The terminal portion of leptospiral immunoglobulin-like protein LigA confers protective immunity against lethal infection in the hamster model of leptospirosis. Vaccine 2007, 25:6277-6286

25. Yan W, Faisal SM, McDonough SP, Divers TJ, Barr SC, Chang CF, Pan MJ, Chang YF: Immunogenicity and protective efficacy of recombinant Leptospira immunoglobulin-like protein B (rLigB) in a hamster challenge model. Microbes Infect 2009, 11:230-237.

26. Picardeau M, Bulach DM, Bouchier C, et al: Genome sequence of the saprophyte Leptospira biflexa provides insights into the evolution of Leptospira and the pathogenesis of leptospirosis. PLOS ONE 2008, 3:e1607.

27. Bourhy P, Frangeul L, Couve E, Glaser P, Saint Girons I, Picardeau M: Complete nucleotide sequence of the LE1 prophage from the spirochete Leptospira biflexa and characterization of its replication and partition functions. J Bacteriol 2005, 187:3931-3940.

28. Poggi D, Oliveira de Giuseppe P, Picardeau M: Antibiotic resistance markers for genetic manipulations of Leptospira spp. Appl Environ Microbiol 2010, 76:4882-4885.

29. Bono JL, Elias AF, Kupko JJ, Stevenson B, Tilly K, Rosa P: Efficient targeted mutagenesis in Borrelia burgdorferi. J Bacteriol 2000, 182:2445-2452.

30. Barocchi MA, Ko Al, Reis MG, McDonald KL, Riley LW: Rapid translocation of polarized MDCK cell monolayers by Leptospira interrogans, an invasive but nonintracellular pathogen. Infect Immun 2002, 70:6926-6932.
31. Cao XJ, Dai J, Xu H, et al: High-coverage proteome analysis reveals the first insight of protein modification systems in the pathogenic spirochete Leptospira interrogans. Cell Res 2010, 20:197-210.

32. Haake DA, Mazel MK, McCoy AM, Milward F, Chao G, Matsunaga J, Wagar EA: Leptospiral outer membrane proteins OmpL1 and LipL41 exhibit synergistic immunoprotection. Infect Immun 1999, 67:6572-6582.

33. Setubal JC, Reis MG, Matsunaga J, Haake DA: Lipoprotein computational prediction in spirochaetal genomes. Microbiology 2006, 152:113-121.

34. Nougayrède JP, Fernandes PJ, Donnenberg MS: Adhesion of enteropathogenic Escherichia coli to host cells. Cell Microbiol 2003, 5:359-372.

35. Pepe JC, Miller VL: Yersinia enterocolitica invasin: a primary role in the initiation of infection. Proc Natl Acad Sci USA 1993, 90:6473-6477.

36. Choy HA, Kelley MM, Croda J, Matsunaga J, Babbitt JT, Ko Al, Picardeau M, Haake DA: The multifunctional LigB adhesin binds homeostatic proteins with potential roles in cutaneous infection by pathogenic Leptospira interrogans. PLoS One 2011, 6:e16879.

37. Atzingen MV, Barbosa AS, De Brito T, Vasconcellos SA, de Morais ZM, Lima DM, Abreu PA, Nascimento AL: Lsa21, a novel leptospiral protein binding adhesive matrix molecules and present during human infection. BMC Microbiol 2008, 8:70.

38. Barbosa AS, Abreu PA, Neves FO, Atzingen MV, Watanabe MM, Vieira ML, Morais ZM, Vasconcellos SA, Nascimento AL: A newly identified leptospiral adhesin mediates attachment to laminin. Infect Immun 2006, 74:6356-6364.

39. Hauk P, Macedo F, Romero EC, Vasconcellos SA, de Morais ZM, Barbosa AS, Ho PL: In LipL32, the major leptospiral lipoprotein, the C terminus is the primary immunogenic domain and mediates interaction with collagen IV and plasma fibronectin. Infect Immun 2008, 76:2642-2650.

40. Longhi MT, Oliveira TR, Romero EC, Gonçales AP, de Morais ZM, Vasconcellos SA, Nascimento AL: A newly identified protein of Leptospira interrogans mediates binding to laminin. J Med Microbiol 2009, 58:1275-1282.

41. Merien F, Truccolo J, Baranton G, Perolat P: Identification of a 36-kDa fibronectin-binding protein expressed by a virulent variant of Leptospira interrogans serovar icterohaemorrhagiae. FEMS Microbiol Lett 2000, 185:17-22.

42. Stevenson B, Choy HA, Pinne M, et al: Leptospira interrogans endostatinlike outer membrane proteins bind host fibronectin, laminin and regulators of complement. PLOS ONE 2007, 2:e1188.

43. Vieira ML, de Morais ZM, Gonçales AP, Romero EC, Vasconcellos SA, Nascimento AL: Lsa63, a newly identified surface protein of Leptospira interrogans binds laminin and collagen IV. J Infect 2010, 60:52-64.

44. Thomas DD, Higbie LM: In vitro association of leptospires with host cells. Infect Immun 1990, 58:581-585.

45. Praetorius J, Spring KR: Specific lectins map the distribution of fibronectin and B-1 integrin on living MDCK cells. Exp Cell Res 2002, 276:52-62.

46. Schoenenberger CA, Zuk A, Zinkl GM, Kendall D, Matlin KS: Integrin expression and localization in normal MDCK cells and transformed MDCK cells lacking apical polarity. J Cell Sci 1994, 107:527-541.

47. Ellinghausen HC, McCullough WG: Nutrition of Leptospira pomona and growth of 13 other serotypes: fractionation of oleic albumin complex and a medium of bovine albumin and polysorbate 80. Am J Vet Res 1965, 26:45-51.

48. Johnson RC, Harris VG: Differentiation of pathogenic and saprophytic leptospires. J Bacteriol 1967, 94:27-31.

49. Bauby H, Saint Girons I, Picardeau M: Construction and complementation of the first auxotrophic mutant in the spirochaete Leptospira meyeri. Microbiology 2003, 149:689-693.

50. Cullen PA, Xu X, Matsunaga J, Sanchez Y, Ko Al, Haake DA, Adler B: Surfaceome of Leptospira spp. Infect Immun 2005, 73:4853-4863.

51. Antoine JC, Jouanne C, Lang T, Prina E, de Chastellier C, Frehel C: Localization of major histocompatibility complex class II molecules in phagolysosomes of murine macrophages infected with Leishmania amazonensis. Infect Immun 1991, 9:764-775.

52. Matsunaga J, Lo M, Bulach DM, Zuerner RL, Adler B, Haake DA: Response of Leptospira interrogans to physiologic osmolarity: relevance in signaling the environment-to-host transition. Infect Immun 2007, 75:2864-2874.

doi:10.1186/1471-2180-11-129

Cite this article as: Figueira et al: Heterologous expression of pathogenspecific genes ligA and ligB in the saprophyte Leptospira biflexa confers enhanced adhesion to cultured cells and fibronectin. BMC Microbiology 2011 11:129. 